\title{
Structure of the Crystalline Complex between Poly- $(\gamma$-methyl L-glutamate) and Chloroform
}

\author{
Shintaro SASAKI and Shigeki TAKIGAWA \\ Department of Polymer Chemistry, Tokyo Institute of Technology, \\ Ookayama, Meguro-ku, Tokyo 152, Japan
}

(Received January 7, 1987)

\begin{abstract}
The crystal structure of the complex between poly $(\gamma$-methyl L-glutamate) (PMLG) and chloroform was studied by X-ray diffraction method. The PMLG main chain takes the right-handed $\alpha$-helical conformation, which is identical with that of the dry form. The unit cell of the complex is hexagonal with lattice dimensions $a=12.7_{5} \AA$ and $c$ (fiber axis) $=26.9 \AA$, and contains eighteen amino-acid residues and about three molecules of chloroform. The complex assumes a statistically disordered structure, where up and down helices are distributed randomly among the lattice sites, and molecules of chloroform locate favorably between antiparallel helices. The side-chain conformation is rather contracted.
\end{abstract}

KEY WORDS Poly $(\gamma$-methyl L-glutamate) / Crystalline Complex / Chloroform / $\alpha$-Helix / Crystal Structure / Statistical Structure / X-Ray Diffraction /

The Pauling-Corey $\alpha$-helical conformation was first discovered for $\operatorname{poly}(\gamma$-methyl Lglutamate) (PMLG). ${ }^{1-5}$ Its various types of films were prepared by casting from different solvents. 6 ,7 The film obtained from the solution in chloroform is highly crystalline, and consists of the right-handed $\alpha$-helices packed in a hexagonal array with the lateral unit-cell dimension $a=11.9 \AA$. At room temperature, three $\alpha$-helical modifications are known, although conformational differences are very small. ${ }^{1-4}$ The helix in the as-cast film contains eighteen amino-acid residues per five turns $(18 / 5$ helix $)$ in an axial repeat distance $c=$ 27.0 $\AA$. A meridional reflection with indices 006 appears at $4.5 \AA$, indicating a departure from the uniform $18 / 5$ helix. $^{1-4}$ The other modifications, obtained by annealing the original form, are a $29 / 8$ helix with $c=43.3 \AA$ and a $69 / 19$ helix with $c=103.0 \AA$, both exhibiting a forbidden meridional reflection at $4.3 \AA$. In a previous paper, ${ }^{8}$ we reported that the lateral dimension varies slightly, depending on the preparation condition, and that above $170^{\circ} \mathrm{C}$ the structure is trigonal with a unit cell containing three helices assuming screw-type motion.

Solutions of PMLG in chloroform were cast conventionally onto glass plates to prepare the film. Liquid-crystalline solutions were stroked onto glass plates to prepare the oriented film. These films on plates were immersed, for instance, in methanol for several hours until they peeled, and subsequently dried. As mentioned below, their X-ray diffraction patterns are characteristic of the $\alpha$-helix. ${ }^{1-5}$ It was recently found that the intact film cast onto a polytetrafluoroethylene plate and peeled without using methanol exhibited additional sharp reflections. ${ }^{9}$ The spacings were invariant for the samples. The new reflections disappeared on immersion of the film in methanol, or on drying at room temperature for a few days. This unstable structure was found to be the 
complex between PMLG and chloroform.

A few complexes between PMLG and lowmolecular-weight compounds were reported in the past. The film prepared from the solution in 1,2-dichloroethane retains the cholesteric twisted structure that is developed in the liquid-crystalline solution. This film forms a crystalline complex with methanol, while the film prepared from the solution in chloroform does not. ${ }^{6}$ The X-ray reflections of the complex were much sharper than those of the original film. There is substantial evidence indicating that in the twisted structure some $\alpha$ helices are closely associated to form a large assembly. This analysis is now in progress. ${ }^{10}$ PMLG forms a complex also with dimethyl phthalate, where a similar helix assembly was proposed. ${ }^{11}$ In this paper, the crystal structure of the complex between PMLG and chloroform was analyzed by X-ray diffraction, and is discussed in comparison with that of the dry form.

\section{EXPERIMENTAL}

A sample of PMLG (Ajicoat A-2000) with viscosity-average molecular weight 110,000 was kindly supplied by Ajinomoto Co., Inc., Japan. The oriented specimen of the complex was prepared on a polytetrafluoroethylene plate by evaporating the solvent chloroform between two electrodes under an electric field of 1500 volt $\mathrm{cm}^{-1}$. It is well known that liquidcrystalline clusters in solutions of synthetic polypeptides are easily aligned in the electric field direction, and that there is no structural difference between the electrically oriented film and the shear oriented one

$\mathrm{X}$-ray diffraction photographs were taken by employing a flat-plate camera and a cylindrical camera with $\mathrm{Cu}-K_{\alpha}$ radiation monochromatized by a graphite monochromator. Reflection spacings were calibrated against reflections of silicon powder sprinkled over the specimen. Reflection intensities were measured from the photographs by using a microphotometer and also by visual comparison with a standard scale, and then corrected for the Lorentz-polarization factor.

\section{RESULTS AND DISCUSSION}

\section{Preliminary Examinations of $X$-Ray Diffraction Patterns}

An endotherm was observed at about $80^{\circ} \mathrm{C}$ on heating the complex at a rate of $10 \mathrm{deg}$ $\min ^{-1}$ by differential scanning calorimetry. The transition was accompanied by a weight loss of about $13 \%$. The diffraction patterns of the complex and the dry sample are shown in

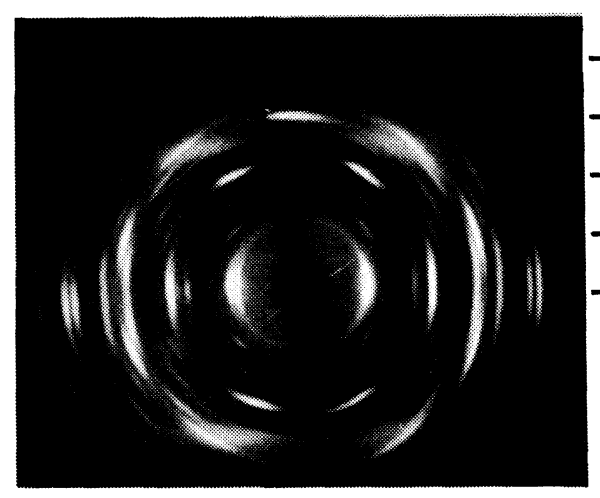

(a)

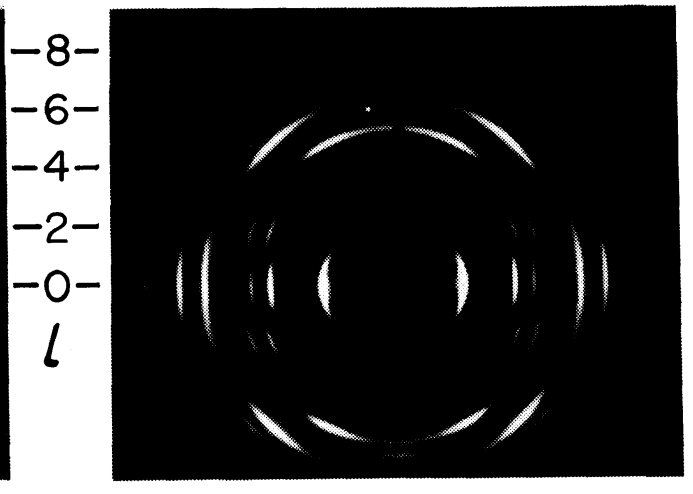

(b)

Figure 1. X-Ray fiber patterns of the complex (a) and the dry sample (b) of PMLG. The orientation direction is vertical. 
Figure 1. The pattern of the latter is typical for PMLG. Both samples exhibit the meridional reflections at $4.5 \AA$ and $1.49_{5} \AA$. The unit height $\left(1.49_{5} \AA\right)$ and the helix pitch $(5.40 \AA)$ are invariant. The complex displayed eleven equatorial and forty non-equatorial independent reflections. The equatorial spacings were explained by a hexagonal unit cell with a dimension $a=12.7_{5} \AA$, which is $7 \%$ larger than that of the dry form.

According to the theory of diffraction by helical molecules, ${ }^{5}$ the orders $(n)$ of the Bessel functions contributing to the $l$ th layer line of the $18 / 5$ helix are selected by

$$
l=5 n+18 m
$$

where $n$ and $m$ are integers as small as possible, since only low-order Bessel functions contribute to intensity. It is established from this relationship that the diffraction pattern of the dry sample is consistent with the $18 / 5$ helix, although the 006 reflection is prohibited for the uniform helix. ${ }^{1-5}$ In contrast, the intensity distribution of the complex does not conform to the $18 / 5$ helix, while it suggests the $6 / 5$ helix, ${ }^{9}$ where three amino-acid residues form an asymmetric unit, and the 006 reflection is inherent.

Since the $\alpha$-helix exhibits polarity along the helix axis, the crystal may consist of both "uppointing" and "down-pointing" helices. If up and down helices locate alternately in the lattice, some reflections suggesting a large unit cell should appear. However, all the reflections observed for the complex as well as for the dry sample were explained by the one-chain unit cell. This offers two possibilities: (1) each crystallite consists of the helices pointing in the same direction, or (2) up and down helices are distributed randomly among the lattice sites. The former is a minor possibility, as was denied for poly(L-alanine). ${ }^{3,4,12}$ The latter is described in terms of the statistical structure in which up and down helices occupy the same lattice site with equal probabilities. The space groups are $\mathrm{P}_{5}$ for the former case and prob- ably $\mathrm{P}_{5} 22$ for the latter.

\section{Stoichiometry}

The diffraction pattern of the complex reappeared when the dry sample was sealed in a glass capillary tube with an amount of chloroform corresponding to $15 \%$ or more of the total sample weight. The samples sealed with chloroform $\leqq 13 \mathrm{wt} \%$ were the mixtures of the complex and the dry form. The weight loss of the complex on drying was about $13 \%$. Since the molecules of chloroform involved in these samples may not exist completely in the crystal lattice, the true amount of complexing chloroform may be slightly less than $13 \mathrm{wt} \%$. The amount of $11.3 \mathrm{wt} \%$ corresponds to the presence of three molecules of chloroform in a unit cell together with eighteen amino-acid residues. The calculated density is plausibly $1.29 \mathrm{~g} \mathrm{~cm}^{-3}$. Since six asymmetric units are required for the hexagonal space groups, the existence probability of chloroform suggests a statistically disordered structure in which about half the sites available for molecules of chloroform are vacant.

\section{Equatorial Projection}

The equatorial intensities of the dry form were elucidated by a smooth curve of the molecular structure factor calculated for the $18 / 5$ helix. ${ }^{1}$ This indicates that the side chains are uniformly arranged about the helix axis, as mentioned below. The considerably different intensity distribution of the complex is attributable to heavy chlorine atoms and deformation of side chains.

The electron-density projection along the $c$ axis is determined by Fourier synthesis in the equatorial plane. The two-dimensional structure is probably centrosymmetric and may have diad axes lying in the plane (the space group p $6 \mathrm{~mm}$ ), where the structure factor are real and assume the same value for pairs of $h k 0$ and $k h 0$ reflections.

The electron density projected on the point $(x, y)$ is defined here by the average of the 
density at $(x, y, z)$ along the $z$ axis over a repeat distance $c$ :

$$
\begin{aligned}
\rho(x y) & \equiv \frac{1}{c} \int_{z=0}^{c} \rho(x y z) \mathrm{d} z \\
& =\frac{18}{V} \sum_{h, k} F(h k 0) \cos \{2 \pi(h x+k y)\}
\end{aligned}
$$

where $V$ is the unit-cell volume and $F(h k 0)$ is the structure factor of the $h k 0$ reflection scaled per amino-acid residue. The structure amplitudes $\left(\left|F_{o}\right|\right)$ are determined in a relative scale from the observed intensity data, while the signs cannot be determined from experiment. The intensity scale factor and the phase factors $(+1$ or -1$)$ can be estimated from a comparison with the calculated structure factors. Although the side-chain conformation is considered to be fairly disordered in the dry form, disregard of the side chains leads to an erroneous scale factor. In this work, the following two models were considered:

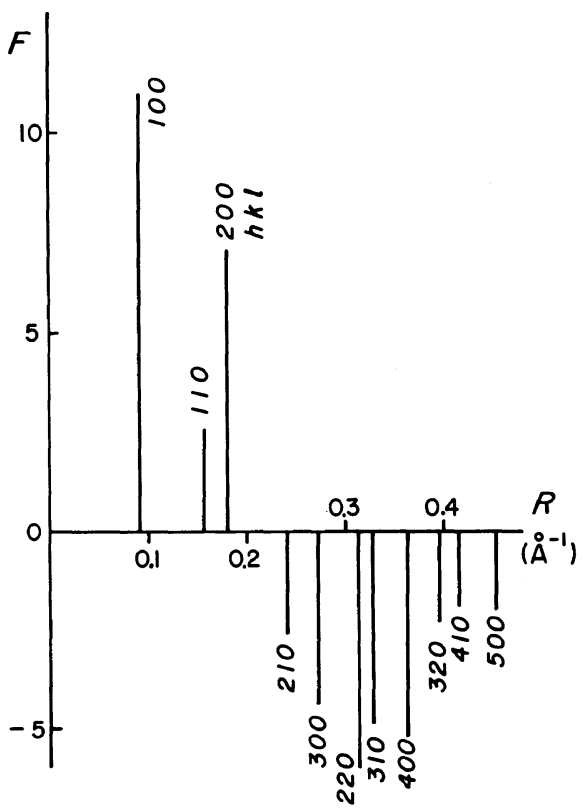

(a)

$\begin{array}{lccccc} & \mathrm{N}-\mathrm{C}^{\alpha}-\mathrm{C}^{\beta}-\mathrm{C}^{\gamma}-\mathrm{C}^{\delta} \mathrm{O}-\mathrm{O}-\mathrm{C} \\ & \chi_{1} & \chi_{2} & \chi_{3} & \chi_{4} \\ \text { model I } & T & T & T & T \\ \text { model II } & \bar{G} & T & G & T\end{array}$

where $T$ is trans (the torsion angle $180^{\circ}$ ), $G$ $\left(60^{\circ}\right)$ and $\bar{G}\left(-60^{\circ}\right)$ are gauche and minus gauche conformations, respectively. The $G$ conformation for $\chi_{1}$ is prohibited from steric hindrance. ${ }^{13-16}$ The side chains are extended in model I, while they are contracted in model II. The atomic coordinates were calculated with the standard dimensions of bond lengths and bond angles. ${ }^{17,18}$ The main-chain torsion angles were set at $\phi\left(\mathrm{N}-\mathrm{C}^{\alpha}\right)=-52.9^{\circ}$, $\psi\left(\mathrm{C}^{\alpha}-\mathrm{C}^{\prime}\right)=-51.5^{\circ}$, and $\omega\left(\mathrm{C}^{\prime}-\mathrm{N}\right)=180.0^{\circ}$.

Since the unit cell contains one helix, and one Bessel function of the lowest order $n$ selected by eq 1 contributes to each layer line, the crystal structure factors of the dry form are approximated by

$F_{h}(R, \psi, l / c)=G_{l}(R) \exp \{\operatorname{in}(\psi+1 / 2 \pi)\}$



(b)

Figure 2. Equatorial structure factors of the complex (a) and the dry form (b) scaled per amino-acid residue. The curve in (b) is the cylindrical molecular structure factor $G_{0}(R)$ calculated for model $\mathrm{I}$. 


$$
G_{l}(R)=\sum_{j} f_{j} J_{n}\left(2 \pi R r_{j}\right) \exp \left\{i\left(-n \phi_{j}+2 \pi l z_{j} / c\right)\right\}
$$

where $R, \psi, l / c$ are cylindrical coordinates in reciprocal space; $J_{n}$ is the $n$th order Bessel function; and $r_{j}, \phi_{j}, z_{j}$ are cylindrical coordinates of the $j$ th atom in a residue. Since $n=0$ for $l=0$, the equatorial structure factors do not depend on $\psi$ and $\phi_{j}$, and are given by

$$
G_{0}(R)=\sum_{j} f_{j} J_{0}\left(2 \pi R r_{j}\right)
$$

In the calculations of structure factors, the

Table I. X-Ray equatorial data of the complex

\begin{tabular}{|c|c|c|}
\hline \multirow{2}{*}{ Index } & \multicolumn{2}{|c|}{ Structure factor, $F_{o}^{\mathrm{a}}$} \\
\hline & Complex & Dry sample \\
\hline 100 & 11.0 & 12.7 \\
\hline 110 & 2.5 & 6.0 \\
\hline 200 & 7.1 & 3.2 \\
\hline 210 & -2.6 & -4.2 \\
\hline 300 & -4.4 & -5.3 \\
\hline 220 & -6.0 & -3.4 \\
\hline 310 & -4.9 & -2.0 \\
\hline 400 & -5.2 & -2.0 \\
\hline 320 & -2.3 & -1.2 \\
\hline 410 & -1.9 & -1.3 \\
\hline 500 & -2.0 & -1.5 \\
\hline 330 & - & -0.9 \\
\hline
\end{tabular}
and the dry form of PMLG

a The structure factors scaled per amino-acid residue.

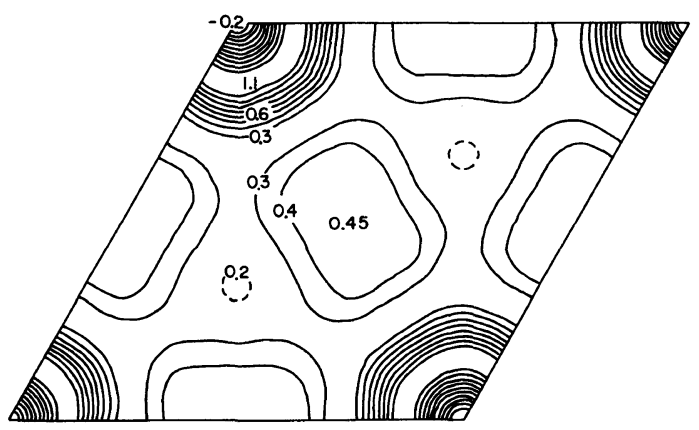

(a) hydrogen atoms where neglected and Debye factors described by the isotropic temperature factos $\left(B_{j}\right)$ were included. Large $B_{j}$ values $\left(\leq 40 \AA^{2}\right.$ ) were assumed for the side-chain atoms. All the $G_{0}(R)$ curves calculated for the above and other selected molecular models exhibited similar features. In Figure $2 b$, the continuous curve represents $G_{0}(R)$ calculated for model I, and the lengths of vertical rods indicate the reflection amplitudes. $G_{0}(R)$ is not sensitive for the side-chain conformation, since it depends only on $r_{j}$. Therefore, a comparison between $\left|F_{o}\right|$ and $G_{0}(R)$ is not to determine the possible side-chain conformation, but the scale factor and the phase factors with the first three amplitudes + and the remainder - .

A similar phase relationship is inferred for the complex. However, the $\left|F_{o}\right|$ data cannot be compared with the cylindrical structure factors because the structure of the complex is not cylindrical. Then, crystal structure factors were calculated by taking into account contributions from both PMLG and chloroform. The molecules of chloroform may locate in the vicinity of the Wyckoff position $2 \mathrm{~b}$ : $(1 / 3,2 / 3)$; $(2 / 3,1 / 3)$; or the position 3c: $(1 / 2,0) ; .(0,1 / 2)$; $(1 / 2,1 / 2)$ in the tow-dimensional space group p6mm. The calculations supported the latter position, and yielded the scale factor and the phase factors, as is shown in Figure 2a. The equatorial data of the complex and the dry form are listed in Table I.

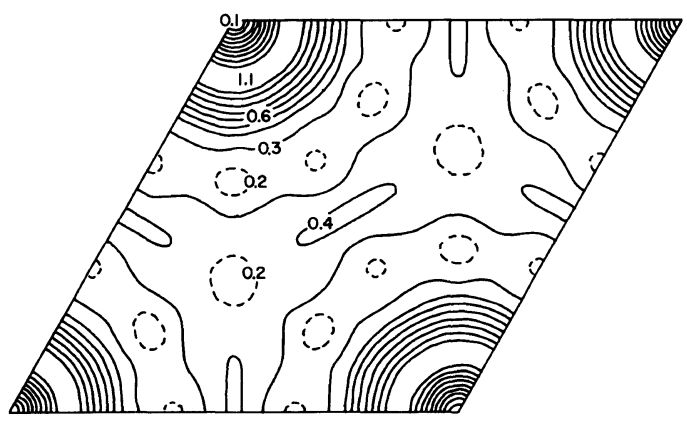

(b)

Figure 3. Electron density maps of the equatorial projection of the complex (a) and the dry form (b). The figures are in units of electrons $\AA^{-3}$. 
The electron-density maps were calculated according to eq 2 , where $F(000)=\sum f_{j}$ was included. As shown in Figure 3, the $\alpha$-helix core is well resolved with the maximum density of 1.1 electrons $\AA^{-3}$. The side chains of the dry form are homogeneously dispersed. The density is condensed between neighboring helices in the complex. This concentration is attributed mainly to chloroform molecules. The density at positions $(1 / 3,2 / 3)$ and $(2 / 3,1 / 3)$ is 0.2 electrons $\AA^{-3}$ for both forms, indicating that the molecules of chloroform do not exist in these regions.

\section{Crystal Structure}

Complexing molecules of chloroform may deform the side chains from the $18 / 5$ helical arrangement. Since it was difficult to depict the manner of deformation, we were forced to assume the $18 / 5$ helical structure for PMLG molecule including the side chains.

The reflections on the strong layer lines of the dry form are strong on the whole. In contrast, the complex exhibits intensity oscillations. On the fifth layer line, for instance, the 105 reflection is weak, the 115 reflection is strong, while the 205 reflection is absent. On the fourth layer line, the 104 reflection is strong, the 114 reflection is absent, while the

Table II. Atomic coordinates and temperature factors of the complex between PMLG and chloroform

\begin{tabular}{clcrrr}
\hline Group & Atom & $x$ & $y$ & $z$ & $B / \AA^{2}$ \\
\hline Amino-acid & $\mathrm{N}$ & 0.123 & 0.003 & 0.012 & 5 \\
residue & $\mathrm{C}^{\alpha}$ & 0.206 & 0.103 & 0.045 & 5 \\
& $\mathrm{C}^{\prime}$ & 0.135 & 0.124 & 0.085 & 5 \\
& $\mathrm{O}$ & 0.161 & 0.128 & 0.130 & 5 \\
& $\mathrm{C}^{\beta}$ & 0.277 & 0.219 & 0.014 & 5 \\
& $\mathrm{C}^{\gamma}$ & 0.350 & 0.201 & -0.027 & 10 \\
& $\mathrm{C}^{\delta}$ & 0.421 & 0.316 & -0.058 & 20 \\
(Carbonyl) & $\mathrm{O}$ & 0.402 & 0.314 & -0.104 & 30 \\
& $\mathrm{O}$ & 0.502 & 0.416 & -0.032 & 30 \\
(Methyl) & $\mathrm{C}$ & 0.561 & 0.516 & -0.065 & 40 \\
Chloroform & $\mathrm{C}$ & 0.520 & 0.045 & 0.0 & 10 \\
& $\mathrm{Cl}$ & 0.550 & -0.055 & 0.038 & 10 \\
& $\mathrm{Cl}$ & 0.650 & 0.145 & -0.038 & 10 \\
& $\mathrm{Cl}$ & 0.390 & -0.055 & -0.038 & 10 \\
\hline
\end{tabular}

204 reflection is moderate. These are due to interference phenomena, possibly, (a) between the $\alpha$-helix core and the side chains, (b) between up and down helices, and (c) between PMLG and chloroform molecules.

The parallel-helix model (space group $\mathrm{P6}_{5}$ ) was examined at first, although this structure is unlikely on account of the strong polarity. On

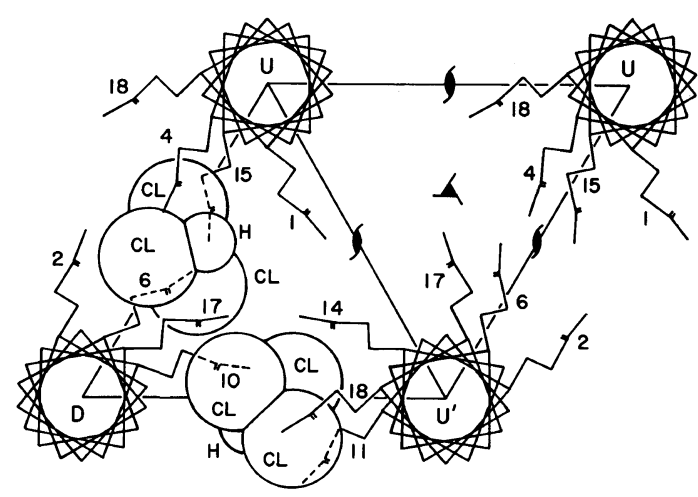

(a)

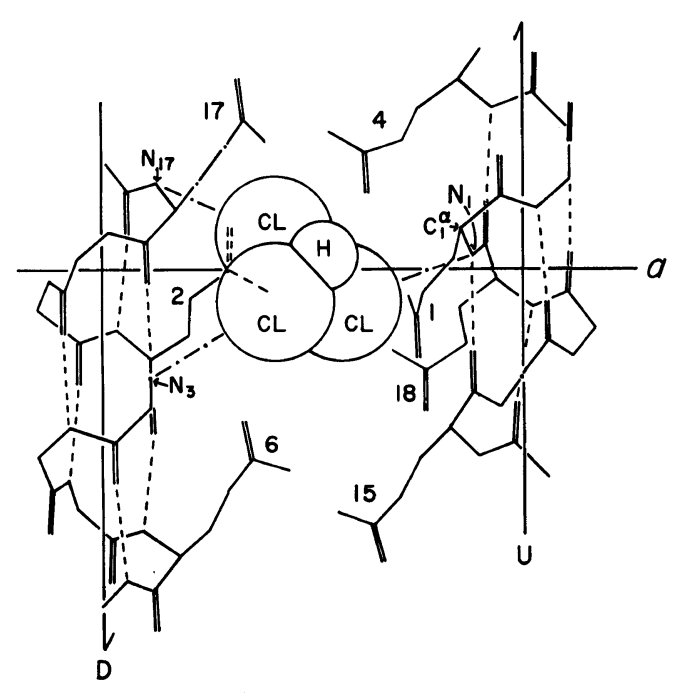

(b)

Figure 4. Crystal structure of the complex between PMLG and chloroform. (a) The projection viewed along the $c$ axis; (b) the arrangement of the chloroform molecule between antiparallel helices. $D$ and $U$ (or $U^{\prime}$ ) denote down and up helices, respectively. The side chain of the $n$th residue is denoted by the number $n$. The methyl groups at the ends of the side chains are omitted for simplicity. 
the basis of the $c$ projection, chloroform molecules were located in the vicinity of the position $(1 / 2,0,0)$ and its related positions. The existence probability was set to 0.5 . The contribution of PMLG helix to the crystal structure factor is provided by $F_{h}$ (eq 3 and 4). The amplitude of $F_{h}$ is given by $\left|G_{l}(R)\right|$, and not affected by the rotation of the helix about the axis and the translation along the axis, although the phase varies. The structure factors were calculated and then compared with the observed data by trial and error, but no aggreement was found. For instance, $\left|G_{5}(R)\right|$ calculated for mode I was large for the reflections 105, 115, and 205. Even by taking into account the effect (c), the observed intensity oscillation cculd not be explained. For model II, $\left|G_{5}(R)\right|$ was small for the 105 reflection, while it was large for the 115 and 205 reflections. It was impossible to enlarge $|F(115)|$ and vanish $|F(205)|$ at the same time.

In the statistical model (space group $\mathrm{P}_{5} 22$ up and down helices are related to each other by the $180^{\circ}$ rotation about the diad axis

Table III. Comparison between observed and calculated structure factors of the complex of PMLG with chloroform

\begin{tabular}{|c|c|c|c|c|c|}
\hline Index & $\left|F_{o}\right|^{\mathrm{a}}$ & $\left|F_{c}\right|^{\mathrm{a}}$ & Index & $\left|F_{o}\right|^{\mathrm{a}}$ & $\left|F_{c}\right|^{\mathrm{a}}$ \\
\hline 100 & 11.0 & 12.5 & 204 & 4.0 & 2.0 \\
\hline 110 & 2.5 & 1.5 & 214 & 3.0 & 0.1 \\
\hline 200 & 7.1 & 6.5 & 304 & - & 1.6 \\
\hline 210 & 2.6 & 2.3 & 105 & 1.8 & 0.4 \\
\hline 300 & 4.4 & 4.3 & 115 & 5.0 & 4.9 \\
\hline 220 & 6.0 & 5.2 & 205 & - & 1.3 \\
\hline 310 & 4.9 & 5.6 & 215 & 3.0 & 1.4 \\
\hline 400 & 5.2 & 5.3 & 305 & 3.4 & 1.6 \\
\hline 320 & 2.3 & 3.2 & 225 & - & 0.7 \\
\hline 410 & 1.9 & 2.3 & 315 & 3.4 & 0.2 \\
\hline 500 & 2.0 & 1.8 & 405 & 3.1 & 1.4 \\
\hline 101 & - & 0.4 & 325 & 2.7 & 1.7 \\
\hline 111 & 2.0 & 1.5 & 006 & $\mathrm{~m}, \sim 2^{\mathrm{b}}$ & 1.6 \\
\hline 2001 & 3.4 & 3.5 & 106 & 1.5 & 0.7 \\
\hline 211 & 3.9 & 3.6 & 116 & 2.5 & 1.1 \\
\hline $\begin{array}{lll}3 & 0 & 1\end{array}$ & 4.0 & 1.9 & 206 & - & 0.8 \\
\hline 102 & 2.4 & 2.6 & 216 & 3.0 & 2.9 \\
\hline 112 & - & 1.3 & 107 & 2.3 & 0.1 \\
\hline 202 & 3.2 & 4.1 & 117 & - & 0.1 \\
\hline 212 & 5.0 & 3.6 & 207 & 3.4 & 0.1 \\
\hline 302 & 2.2 & 2.1 & 217 & 2.6 & 0.7 \\
\hline 222 & 3.6 & 2.7 & 108 & 2.0 & 0.2 \\
\hline 103 & 2.2 & 0.9 & 118 & 2.9 & 1.8 \\
\hline 113 & - & 0.3 & 208 & - & 0.2 \\
\hline 203 & 3.4 & 0.5 & 218 & 3.0 & 0.9 \\
\hline 213 & 2.6 & 1.8 & 109 & - & 0.7 \\
\hline $\begin{array}{lll}3 & 0 & 3\end{array}$ & 4.0 & 1.7 & 119 & 2.5 & 0.2 \\
\hline 223 & - & 1.4 & 209 & - & 0.1 \\
\hline 313 & 3.1 & 0.7 & 219 & 2.0 & 0.8 \\
\hline 403 & 4.4 & 2.3 & $\begin{array}{lll}1 & 0 & 10\end{array}$ & - & 1.4 \\
\hline 323 & 3.0 & 2.9 & 1110 & 2.6 & 2.5 \\
\hline 104 & 4.2 & 3.3 & $\begin{array}{lll}0 & 0 & 18\end{array}$ & $\mathrm{~s}, \sim 3^{\mathrm{b}}$ & 2.6 \\
\hline 114 & - & 0.5 & & & \\
\hline
\end{tabular}

a The structure factors are scaled per amino-acid residue.

b The intensity measurements of the meridional reflections were not accurate. Code: m, moderate; s, strong. 
coinciding with the $a$ axis. This disorder should give rise to diffuse layer-line streaks, which actually appeared on non-equatorial layer lines. As a result of the interference effect (b), $\left|G_{l}(R)\right|$ for the Bragg reflections is given by the real (cosine) term of eq $4 .^{3,4}$ The imaginary (sine) term contributes to the intensities of the diffuse streaks. By similar considerations to the case of $\mathrm{P} 6_{5}$, model I was denied, while model II was found to be prospective. A rather good agreement between observed $\left(\left|F_{o}\right|\right)$ and calculated $\left(\left|F_{c}\right|\right)$ structure amplitudes was attained for model II after rotational and translational adjustments of PMLG and chloroform molecules. The molecules of chloroform may rotate at room temperature, but the spherical averaging of the density lessened the agreement. The fractional atomic coordinates and the assumed temperature factors are listed in Table II. The comparison between

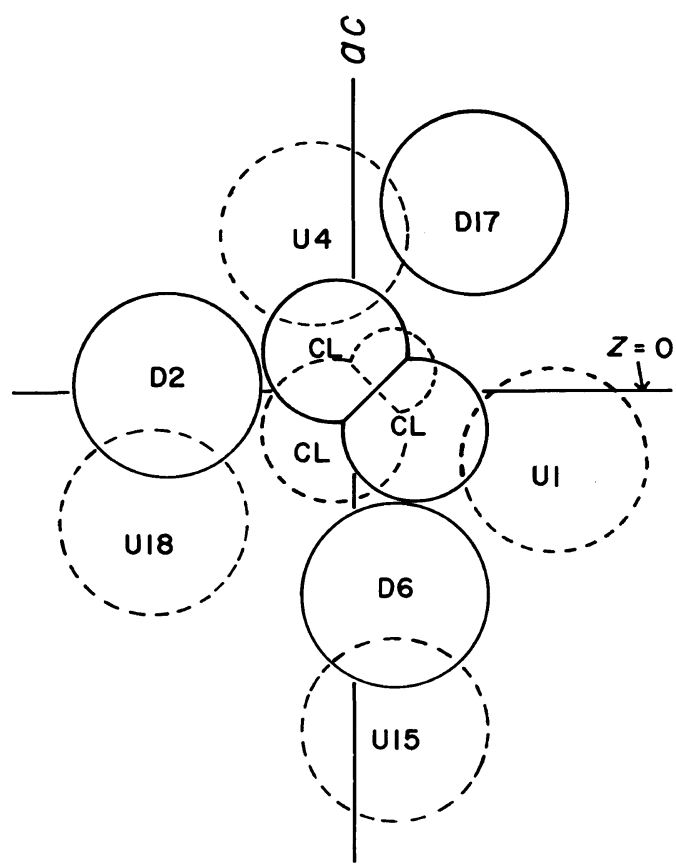

(a)
$\left|F_{o}\right|$ and $\left|F_{c}\right|$ is given in Table III. The discrepancy factor $R=\sum\left\{\left|F_{o}\right|-\left|F_{c}\right|\right\} / \sum\left|F_{o}\right|$ is 0.35 for the observed reflections.

The crystal structure is schematically drawn in Figure 4. Although up and down helices are superimposed for each lattice site, an up (denoted by $U$ or $U^{\prime}$ ) or down (D) helix is illustrated for simplicity. Chloroform molecules are drawn between antiparallel helices. The packing features are discussed below.

The real structure is considered to be more complicated; the helix is not $18 / 5$ but $6 / 5$, and the side-chain conformation may not be unique. Although the discrepancy factor is not small enough at the present stage, further refinement was judged to be uncertain.

\section{Packing Features of Chloroform Molecules}

The possible manner of packing of chloroform molecules is illustrated in Fgiure 5 for

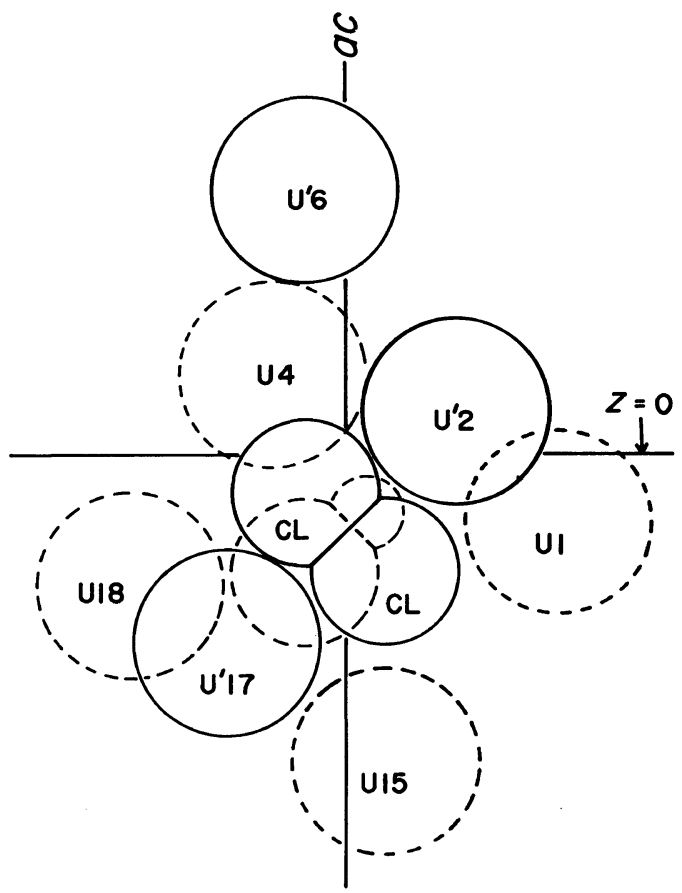

(b)

Figure 5. Packing features of chloroform molecules between antiparallel helices (a) and between parallel helices (b), viewed along the $a$ axis. D $n$, Un, and $\mathrm{U}^{\prime} n$ denote the side chains of the $n$th residues of $\mathrm{D}, \mathrm{U}$, and $\mathrm{U}^{\prime}$ helices, respectively. The circles illustrate the cross sections of the side chains each with a diameter of $4.8 \AA$. 
two cases: (a) between antiparallel helices and (b) between parallel helices. Figure $5 \mathrm{a}$ is the pattern viewed along the $a$ axis in the direction from the down helix (D) to the up helix (U) in Figure 4. Figure $5 b$ is viewed in the direction from $U^{\prime}$ to $U$. On the assumption of the uniform $18 / 5$ helix with the unique side-chain conformation (model II), some short interatomic contacts are inevitable. Slight displacements of side chains allow chloroform molecules to fit into neighboring helices.

The molecules of chloroform can be incorporated closely to the $a$ axis between antiparallel helices (Figures 4 and 5a). Therefore, it is closely superimposed to the chloroform molecule that is related by the $180^{\circ}$ rotation about the $a$ axis. This arrangement yielded good agreement between $\left|F_{o}\right|$ and $\left|F_{c}\right|$ (Table III). The arrangement of the chloform molecule between parallel helices shown in Figure $5 b$ was denied by the structure factor calculations. Therefore, molecules of chloroform may exist favorably between stastically paired antiparallel helices. This provides an explanation for the existence probability of chloroform to be about 0.5 .

The orientation of the chloroform molecule between antiparallel helices (Figure 4a) may permit the coordination of nitrogen atoms to chlorine atoms. The complex exhibited the infrared absorption band associated with the $\mathrm{C}-\mathrm{Cl}$ stretching vibration at $755 \mathrm{~cm}^{-1}$. This wave number and the shape of the band coincide with those of the pure solvent. The infrared absorptions of PMLG did not change by the complex formation. Accordingly, there is no special interactions between PMLG and chloroform, and the complex is produced by van der Waals forces.

\section{CONCLUSIONS}

A crystalline complex was found between chloroform and PMLG with the $\alpha$-helical conformation. The transition from the complex to the dry form and the reverse were repro- ducible.

We may summarize the present results on the structure of the complex as follows.

(1) The unit cell is hexagonal (space group $\mathrm{P}_{5} 22$ ) with lattice dimensions $a=12.7_{5} \AA$ and $c$ (fiber axis) $=26.9 \AA$. and contains eighteen amino-acid residues and about three molecules of chloroform.

(2) The molecules of chloroform locate in the vicinity of the twofold screw axes between neighboring helices, instead of the threefold screw axes surrounded by three helices.

(3) The crystal structure is statistically disordered in the following two respects: (a) up and down helices occupy the same lattice site with equal probabilities, and (b) molecules of chloroform exist favorably between antiparallel helices.

(4) The side chains are not fully extended, but rather contracted.

Since the original complex has a statistical structure with respect to the helix direction, the dry form must hold the same disorder. The complex exhibits the 006 meridional reflection at $4.5 \AA$ inherently. The dry form also exhibits this reflection. It suggests that the $6 / 5$ helical features of the complex are somehow retained in the dry form.

There is no experimental evidence indicating special interactions between PMLG and chloroform molecules. The complex is formed essentially by van der Waals interactions. The size of the side chain of PMLG is thin, while the roots of the side chains are constrained by the $\alpha$-helix core. Therefore, it seems likely that rearrangements and/or aggregations of the side chains offer room for chloroform. It is not understandable as yet why the molecules of chloroform do not locate in the vicinity of the threefold screw axes surrounded by three helices in spite of the favorable opportunity in view of steric factors.

\section{REFERENCES}

1. C. H. Bamford, L. Brown, A. Elliot, W. E. Hanby, and I. F. Trotter, Nature (London), 169, 357 (1952); 


\section{S. SASAKI and S. TAKIGAWA}

Proc. R. Soc. London, Ser. B, 141, 49 (1953).

2. L. Brown and I. F. Trotter, Trans. Faraday Soc, 52 , 537 (1956).

3. A. Elliott, "Poly- $\alpha$-Amino Acids," G. D. Fasman, Ed., Marcel Dekker, New York, N. Y., 1967, pp 137.

4. R. D. B. Fraser and T. P. MacRae, "Conformation in Fibrous Proteins," Academic Press, New York, 1973.

5. W. Cochran, F. H. C. Crick, and V. Vand, Acta Crystallogr., 5, 581 (1952).

6. J. Watanabe, S. Sasaki, and I. Uematsu, Polym. J., 9, 337 (1977).

7. K. Ito, T. Kajiyama, and M. Takayanagi, Polym. J., 9, 355 (1977).

8. S. Sasaki and I. Uematsu, J. Polym. Sci., Polym. Phys. Ed., 23, 263 (1985).

9. S. Sasaki, J. Polym. Sci., Polym. Phys. Ed., in press.
10. S. Sasaki, to appear.

11. J. Watanabe, Y. Sasanuma, A. Endo, and I. Uematsu, Polymer, 25, 698 (1984).

12. S. Arnott and A. J. Wonacott, J. Mol. Biol., 21, 371 (1966).

13. K. Itoh and T. Shimanouchi, Biopolymers, 9, 383 (1970).

14. A. Tanaka and Y. Ishida, J. Polym. Sci., Polym. Phys. Ed., 11, 1117 (1973).

15. K. Nagayama, Seibutsu Butsuri, 16, 68 (1976).

16. A. Tsutsumi, B. Perly, A. Forchioni, and C. Chachaty, Macromolecules, 11, 977 (1978).

17. R. B. Corey and L. Pauling, Proc. R. Soc. London, Ser. B, 141, 10 (1953).

18. "International Tables for X-ray Crystallography," Vol. III, Kynoch Press, Birmingham, England, 1968, Chapter 4. 